\title{
COMPACT LIE GROUPS WHICH ACT ON EUCLIDEAN SPACE WITHOUT FIXED POINTS
}

\author{
ALLAN L. EDMONDS ${ }^{1}$ AND RONNIE LEE ${ }^{2}$
}

\begin{abstract}
It is shown that a compact Lie group $G$ with identity component $G_{0}$ acts without fixed points on euclidean space if and only if $G_{0}$ is nonabelian or $G / G_{0}$ is not of prime power order, which completes earlier work of P. E. Conner and E. E. Floyd, Conner and D. Montgomery, and W.C. Hsiang and W.-Y. Hsiang.
\end{abstract}

In this note we describe completely the class of compact Lie groups which can act without fixed points on euclidean space, thus completing earlier work of P. E. Conner and E. E. Floyd [2], followed by that of Conner and D. Montgomery [3] and W.-C. Hsiang and W.-Y. Hsiang [4, 1.9] who extended Conner and Floyd's original idea.

THEOREM. Let $G$ be a compact Lie group with identity component $G_{0}$. Then $G$ acts without fixed points on some euclidean space if and only if $G_{0}$ is nonabelian or $G / G_{0}$ does not have prime power order.

If $G_{0}$ is abelian and $G / G_{0}$ has prime power order $p^{k}$ and $G$ acts on $\mathbf{R}^{n}$, then by P. A. Smith theory the fixed point set $\left(\mathbf{R}_{n}\right)^{G_{0}}$ is nonempty and $\mathbf{Z}$-acyclic. Then $\left(\mathbf{R}^{n}\right)^{G_{0}}$ is a $G / G_{0^{-}}$space and $\left(\mathbf{R}^{n}\right)^{G}=\left[\left(\mathbf{R}^{n}\right)^{G_{0}}\right]^{G / G_{0}}$ is nonempty and $\mathbf{Z}_{p^{-}}$ acyclic, again by Smith theory.

We now begin to consider the converse. The following is a fairly well-known topological analogue of induced representations.

INDUCTION LeMMA. If $G$ is a compact Lie group, $H \subset G$ is a subgroup of finite index $k$, and $X$ is an $H$-space, then there is an action of $G$ on $X^{k}$, the product of $k$ copies of $X$, such that the fixed point set $\left(X^{k}\right)^{G}$ is the diagonal copy of $X^{H}$. Further, if $f: X \rightarrow X$ is an H-map, then the natural map $f^{k}: X^{k} \rightarrow X^{k}$ is a Gmap.

We indicate a proof, following a suggestion of G. E. Bredon. See also [6, 1.2].

Notice that, by choosing coset representatives for $H$ in $G, X^{k}$ can be identified with Maps ${ }^{H}(G, X)$, the set of $H$-maps from $G$ to $X$, where $H$ acts on $G$ by left translation. Then $G$ acts on $\operatorname{Maps}^{H}(G, X)$ by $(g \varphi)\left(g^{\prime}\right)=\varphi\left(g^{\prime} g\right)$, with fixed point set $\left[\operatorname{Maps}^{H}(G, X)\right]^{G} \cong X^{H}$, via the map $\varphi \mapsto \varphi(e)$, where $e$ is the identity element in $G$. If $f: X \rightarrow X$ is an $H$-map, then $f^{k}: X^{k} \rightarrow X^{k}$ is

Received by the editors January 10, 1975 and, in revised form, May 16, 1975.

AMS (MOS) subject classifications (1970). Primary 57E25, 57E10.

1 Supported in part by NSF grants GP-33031X and GP-36418X1.

2 Supported in part by the National Science Foundation and the Alfred P. Sloan Foundation. 
identified with $f_{*}: \operatorname{Maps}^{H}(G, X) \rightarrow \operatorname{Maps}^{H}(G, X)$, where $f_{*}(\varphi)=f \circ \varphi$ and is easily seen to be a $G$-map.

Now, returning to the proof of the Theorem, if $G_{0} \subset G$ is nonabelian then, according to $[4,1.9], G_{0}$ acts without fixed points on some euclidean space; hence the Induction Lemma implies that $G$ does also. Similarly, if $G / G_{0}$ acts without fixed points on euclidean space, then $G$ does also, via the homomorphism $G \rightarrow G / G_{0}$. ( If an effective action is desired simply cross with any effective representation of $G$.) Thus the proof of the Theorem is reduced to the following result.

Proposition. If $G$ is a finite group not of prime power order, then $G$ acts without fixed points on some euclidean space.

The method of Conner and Floyd [2] as refined by J. M. Kister [5] shows that it suffices to find a sphere $S$ on which $G$ acts simplicially and without fixed points and a simplicial $G$-map $f: S \rightarrow S$ of degree 0 . For one can then form the infinite mapping telescope $T$ associated to $f$, a contractible simplicial complex on which $G$ acts without fixed points. One then embeds $T$ in a representation of $G$. An open invariant regular neighborhood of $T$ finally provides the desired action.

The proof of the Proposition proceeds by induction on the order of $G$. We may assume, using the Induction Lemma, that every proper subgroup of $G$ has prime power order. Then by an elementary result of group theory [7, 6.5.7] $G$ must be solvable. Therefore we may find a surjection $G \rightarrow \mathbf{Z}_{p}$ and an injection $\mathbf{Z}_{q} \rightarrow G$ where $p$ and $q$ are distinct primes and $\mathbf{Z}_{p}$ and $\mathbf{Z}_{q}$ denote the cyclic groups of orders $p$ and $q$, respectively.

Let $D_{p}$ and $D_{q}$ be standard 2-disks with boundary circles $S_{p}$ and $S_{q}$ on which $\mathbf{Z}_{p}$ and $\mathbf{Z}_{q}$ act by rotations. The surjection $G \rightarrow \mathbf{Z}_{p}$ makes $D_{p}$ into a $G$-space with one fixed point and the Induction Lemma shows that the $2 k$-disk $\left(D_{q}\right)^{k}$ is a $G$-space with one fixed point, where $k$ is the index of $\mathbf{Z}_{q}$ in $G$. Let $S_{q}^{2 k-1}$ denote the boundary $(2 k-1)$-sphere, on which $G$ acts without fixed points and consider the join $S=S_{p} * S_{q}^{2 k-1}$, a $(2 k+1)$-sphere on which $G$ acts without fixed points in the obvious way. We claim that $S$ admits a simplicial $G$-map of degree 0 .

To construct the desired $G$-map, begin by noticing that there are equivariant maps $\alpha: S_{p} \rightarrow S_{p}$ and $\beta: S_{q} \rightarrow S_{q}$ having degrees $1+m p$ and $1+n q$, respectively, where $m$ and $n$ are arbitrary integers. (E.g., $\alpha(z)=z^{1+m p}$, where $\mathbf{Z}_{p}$ acts on $S_{p}$ by multiplication by $\exp (2 \pi i / p)$.) Let $c \beta: D_{q} \rightarrow D_{q}$ be the obvious conical extension of $\beta$ to all of $D_{q}$.

Now $\alpha$ is also a $G$-map, as is the map $(c \beta)^{k}:\left(D_{q}\right)^{k} \rightarrow\left(D_{q}\right)^{k}$. Let $\gamma: S_{q}^{2 k-1}$ $\rightarrow S_{q}^{2 k-1}$ be the restriction of $(c \beta)^{k}$ to the boundary. Notice that degree $(\gamma)$ $=(1+n q)^{k}$.

In his discussion of the Conner and Floyd construction, Bredon [1, pp. 58-62] shows how to construct, given $G$-maps such as $\alpha$ and $\gamma$, another simplicial $G$-map $\alpha \square \gamma: S_{p} * S_{q}^{2 k-1} \rightarrow S_{p} * S_{q}^{2 k-1}$ such that degree $(\alpha \square \gamma)$ $=\operatorname{degree}(\alpha)+\operatorname{degree}(\gamma)-1$. Thus degree $(\alpha \square \gamma)=m p+(1+n q)^{k}$. Since $(p, q)=1$ we can choose integers $n$ and $l$ such that $1+n q=l p$. Then degree $(\alpha \square \gamma)=m p+(l p)^{k}$, where $m$ is still an arbitrary integer. Let $m$ $=-l^{k} p^{k-1}$. Then degree $(\alpha \square \gamma)=0$ as desired. 


\section{REFERENCES}

1. G. E. Bredon, Introduction to compact transformation groups, Academic Press, New York, 1972.

2. P. E. Conner and E. E. Floyd, On the construction of periodic maps without fixed points, Proc. Amer. Math. Soc. 10 (1959), 354-360. MR 21 \#3860.

3. P. E. Conner and D. Montogomery, An example for $S O(3)$, Proc. Nat. Acad. Sci. U.S.A. 48 (1962), 1918-1922. MR 26 \#6300.

4. W. C. Hsiang and W. Y. Hsiang, Differentiable actions of compact connected classical groups. I, Amer. J. Math. 89 (1967), 705-786. MR 36 \#304.

5. J. M. Kister, Differentiable periodic actions on $E^{8}$ without fixed points, Amer. J. Math. 85 (1963), 316-319. MR 27 \#4227.

6. R. A. Oliver, Smooth fixed point free actions of compact Lie groups on disks, Thesis, Princeton University, 1974.

7. W. R. Scott, Group theory, Prentice-Hall, Englewood Cliffs, N.J., 1964. MR 29 \#4785.

Department of Mathematics, Cornell University, Ithaca, New York 14850

Department of Mathematics, Yale University, New Haven, Connecticut 06520 